\title{
Self vs. Parent: Factors Influencing Likelihood of Hiring a
}

\section{Healthcare Advocate}

\author{
April C. May ${ }^{1,2^{*}}$, Danielle Casteel ${ }^{1}$, Symone A. McKinnon ${ }^{1} \&$ Terry A. Cronan ${ }^{1,2}$ \\ ${ }^{1}$ Department of Psychology, San Diego State University, USA \\ 2 Joint Doctoral Program in Clinical Psychology, University of California, San Diego/San Diego State \\ University, USA \\ *April C. May, E-mail: aprilmay32@gmail.com
}

Received: February 24, 2017

Accepted: March 5, 2017

Online Published: June 17, 2017

doi:10.22158/rhs.v2n3p247

URL: http://dx.doi.org/10.22158/rhs.v2n3p247

\begin{abstract}
Objective: To determine how the factors that may lead an individual to hire a healthcare advocate to aid him/herself in navigating the healthcare system when dealing with chronic or complex health issues differ from the factors that are considered when deciding to hire a healthcare advocate for one's parent.
\end{abstract}

Methods: 1,740 randomly selected participants completed a brief vignette-based questionnaire that indicated their likelihood of hiring an HCA for oneself or a parent. Confirmatory factor analysis and structural equation modeling were used to test the effects of predisposing, enabling, and illness factors on the predicted likelihood.

Results: Although neither model fit well statistically, both fit well descriptively. The direct path from predisposing to enabling factors and the indirect path from predisposing factors to illness level were significant in both models.

Discussion: Understanding the factors that influence the decision to hire an HCA could help health providers target patients who are most likely to use HCA services, thereby reducing the burden on the healthcare system and improving quality of care.

\section{Keywords}

Healthcare Advocates, health services, chronic conditions

\section{Introduction}

The healthcare system faces increasing challenges in delivering high quality care because of the growing prevalence of chronic conditions (Bodenheimer, 2008; Schoen et al., 2007; Singer et al., 2011). Patients with chronic conditions often require multiple practitioners, and coordinated care is critical for effective treatment (Fani Marvasti \& Stafford, 2012; Schoen et al., 2007; Singer et al., 2011). However, because the healthcare system in the United States is better designed for handling acute illnesses than 
for chronic illnesses (Wagner et al., 2001), patients with chronic illnesses often receive suboptimal care and are more likely to experience medical errors, poor health outcomes, and dissatisfaction with their care (Boyd et al., 2007; Fani Marvasti \& Stafford, 2012; Siu, Spragens, Inouye, Morrison, \& Leff, 2009). Poor communication between patients and physicians can lead to difficulties in establishing treatment plans and result in poor adherence and worsening health among the patients (Parekh \& Barton, 2010). There are often communication difficulties, not only between patients and physicians, but also between healthcare professionals because of insufficient information and delayed responses; this can result in poor adherence and worsening health among the patients (Vogeli et al., 2007). These difficulties with communication are reported as one of the most common reasons for patient dissatisfaction with healthcare (Cousin, Schmid Mast, Roter, \& Hall, 2012; Wright, Sparks, \& O'Hair, 2012). To improve communication and better coordinate care, there has been a shift toward more patient involvement in medical decision-making.

Patients' involvement in their healthcare has been termed "shared decision making" (Elwyn et al., 2012) and ensures that patients understand their conditions, the best treatment options available, and potential health outcomes and risks (Elwyn et al., 2012; Kaplan, 2004). Patients who are better informed and more involved in their healthcare are more likely to follow their treatment plans, be more satisfied with their care, and have improved health outcomes (Robinson \& Thomson, 2001). However, not all patients are mentally and physically able, or feel confident and knowledgeable enough, to navigate the healthcare system and make important medical decisions (Pickard \& Knight, 2005). Thus, over 34.2 million adults in the United States have been a caregiver to someone age 50 or older within the past 12 months (Family Caregiver Alliance, 2012). Healthcare Advocates (HCAs) are one resource that could help reduce the caregiving burden placed on family members, help individuals navigate their own healthcare options, and improve quality of life for the patient.

Healthcare advocacy was created as an avenue for patients to receive help navigating the healthcare system by providing liaisons to bridge the gap between patients and their healthcare teams (Carlson et al., 2011). HCAs guide patients through the healthcare system and represent the interests of the patients to ensure the best possible care (Hurst, 2007; Schwartz, 2002). HCAs can provide a variety of services, including accompanying patients to doctors' visits, staying with a patient in the hospital overnight, providing emotional support and encouragement, scheduling appointments, assisting with insurance issues, and researching treatment options for the patient (Carlson et al., 2011). The services provided by HCAs increase patient satisfaction and quality of care and reduce unnecessary medical costs (Wilber, Allen, Shannon, \& Alongi, 2003). Although HCAs have been shown to be effective, patients still underuse them. Previous researchers have found that reduced confidence in the healthcare system and the severity of a health condition increased an individual's perceived likelihood of hiring an HCA (Cronan et al., 2010; Van Liew \& Cronan, 2012). Identifying additional factors that contribute to an individual's likelihood of hiring an HCA may help target patients who could benefit the most from their use. 
In 1973, Anderson and Newman presented a theoretical framework for viewing healthcare utilization (see Figure 1). Within this framework, factors that contribute to healthcare use are classified into three categories: predisposing, enabling, and illness factors.

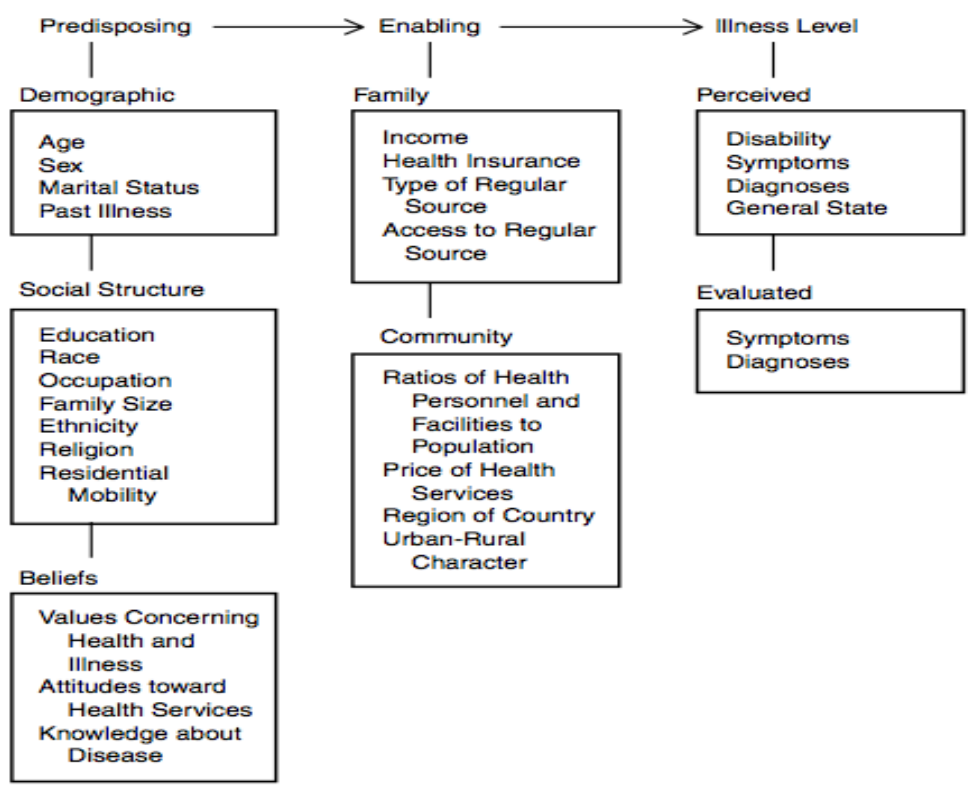

Figure 1. Individual Determinants of Health Service Use, as Adapted from the Andersen-Newman Model of Healthcare Use

Predisposing factors include individual characteristics that exist prior to any diagnosis, such as age, gender and ethnicity. For example, older individuals report substantially higher monthly healthcare expenditures than younger individuals (Yang, Norton, \& Stearns, 2003). Factors affecting individuals' lifestyles, such as ethnicity, may also relate to the use of health services. Specifically, minority ethnic groups, especially African Americans and Hispanics, tend to use healthcare services less than Caucasians (Gaskin, Dinwiddie, Chan, \& McCleary, 2011). Thus, these predisposing sociodemographic factors may influence an individual's likelihood of using healthcare services.

The enabling component of the model is an evaluation of an individual's status and the resources available to seek and receive health services. This component is comprised of resource variables, including insurance coverage, income, and education level. For example, income is related to health and care seeking; people with lower income consistently show higher rates of poor health behaviors, while those with higher income are often more able to obtain healthcare (Hu \& Stowe, 2013; Schoen et al., 2007). People with higher levels of education are also more likely to practice healthy behaviors, attend regular appointments with their physician, and are less likely to require high cost emergency services than those with less education (Lodi-Smith et al., 2010; Schoen et al., 2007). These enabling variables contribute to individual differences in the attainability of healthcare. 
The illness component is the final component in predicting health service use and is the most immediate cause of healthcare use. Within the theoretical framework, the individual or family must have an illness that requires services, or must perceive a high probability of illness occurrence, to seek services (Andersen \& Newman, 1973). Both perceived and evaluated health status are leading causes of healthcare use. People with an illness, or those who perceive their health as poor, report a higher number of physician visits, hospital visits, and prescription medications, than those who perceive themselves as healthy (Andersen \& Newman, 1973; Frostholm et al., 2005).

Anderson and Newman's model of healthcare utilization has broad applications to the understanding of general healthcare use, as well as the use of social services, nursing homes, and other types of caretakers (Aday \& Awe, 1997; Andersen \& Newman, 1973; Gelberg, Andersen, \& Leake, 2000). However, it is important to note that this theoretical model of healthcare use and the factors involved addresses only individual determinants. In many cases, however, it is not the individual who determines the need for health services. Proxy reports provided by a family member, often the child of the patient, are frequently used as a measure of the health and well-being of older individuals (Schulz et al., 2013). Proxy reports are used most often when the individual's condition is severe and his or her health is very poor, or when a person has dementia (Neumann et al., 2000). Considering that a caregiver is often the one involved with medical decision-making, it is important to know how well a proxy report provided by a family member matches the health status of the person of interest, and what may influence a caregiver's likelihood of hiring an HCA for another person. In the present study proxy ratings were examined to determine how this might translate to differences between family members and individuals in the predicted likelihood of using healthcare services, such as those of an HCA.

Although researchers have investigated specific factors that may increase an individual's likelihood of using an HCA, it has yet to be determined how consistent the responses are when considering services for oneself versus for a parent. A clearer understanding of a person's likelihood of hiring a healthcare advocate for themselves, as well as for their parents, may help organizations and researchers design and implement health advocacy services to target patients who will be interested in and benefit the most from these types of services. The present study is intended to compare the model fit of an adaptation of the Anderson-Newman healthcare use model for application to individuals and to parents. To investigate the influence of these factors on an individual's likelihood of hiring an HCA, two main hypotheses were tested: (1) predisposing factors are directly related to enabling factors, and enabling factors are directly related to illness level, which has a direct effect on the likelihood of hiring a healthcare advocate; (2) the overall model depicted in Figure 2 will fit, both statistically and descriptively. The application of the model to predicting the likelihood of hiring an HCA was compared for oneself and a parent to determine whether it is applicable to decisions made by caregivers. 


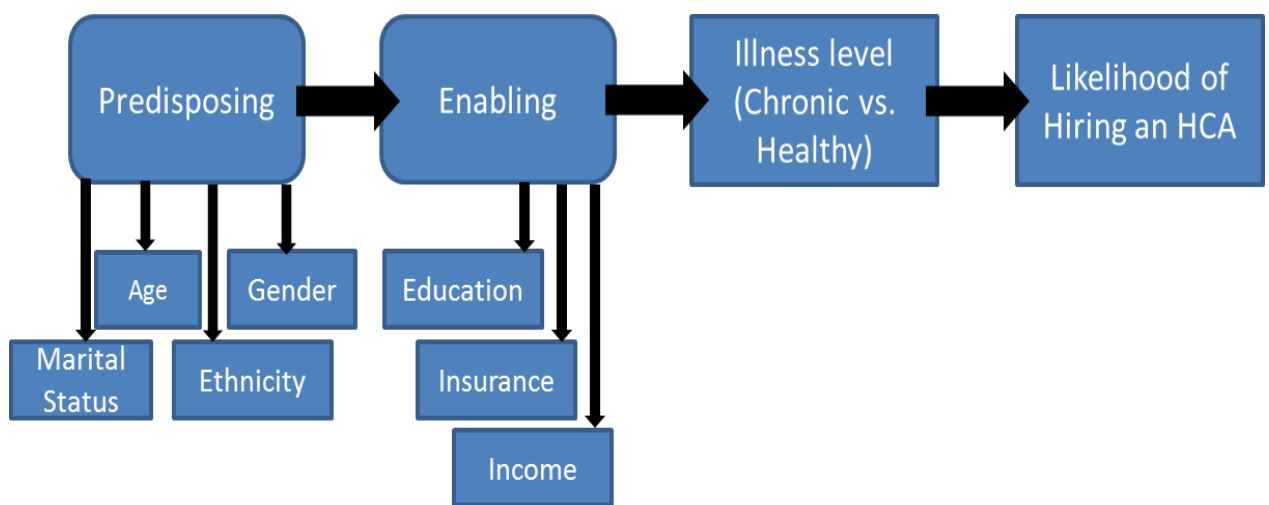

Figure 2. Predicted Model of the Likelihood of Hiring a Healthcare Advocate. The Predisposing Factor is Comprised of Gender, Ethnicity, Age, and Relationship Status. The Enabling Factor is Comprised of Annual Income, Insurance Coverage, and Education Level

\section{Method}

The data for this study were collected from two separate studies. In the first study participants were asked to complete a vignette-based questionnaire on their perceived likelihood of hiring an HCA for a parent, should their parent become ill or injured. In the second study, with the same format, participants were asked about their own perceived likelihood of hiring an HCA if they were to become ill or injured. Participants were 1,740 adults $(\mathrm{N}=1090$ for study $1 ; \mathrm{N}=650$ for study 2 ) randomly selected from the community. Participants had to be 18 years or older to participate, and able to speak and read English. The mean participant age was 44.9 years $(S D=17.17) ; 52.9 \%$ of the participants were female, $69.4 \%$ were Caucasian, $60.1 \%$ had a bachelor's degree or higher, $56.0 \%$ earned less than $\$ 90,000$ per year, and the majority of participants reported having health insurance (85.5\%). Most participants reported having a good understanding of the concept of an HCA ( $M=7.5$ on a 10-point scale, $S D=2.2)$. See Table 1 for detailed demographic characteristics of the two samples.

Table 1. Sample Demographics and Descriptive Statistics

\begin{tabular}{lll}
\hline & Self $(\mathbf{n}=\mathbf{6 5 0})$ & Parent $(\mathbf{n}=\mathbf{1 0 9 0})$ \\
\hline Predisposing Factors & & \\
Age & & \\
Mean & 46.01 & 43.75 \\
SD & 17.17 & 16.77 \\
Gender (\%) & & \\
Male & 42.3 & 47.0 \\
Female & 57.7 & 53.0 \\
Ethnicity (\%) & & \\
Caucasian & 74.4 & 68.2 \\
Minority & 25.6 & 31.8 \\
\hline
\end{tabular}




\begin{tabular}{lrc} 
Marital Status (\%) & 30.1 & 37.3 \\
Single & 60.7 & 51.6 \\
Married/Domestic Partner & 9.1 & 11.1 \\
Divorced/Separated/Widowed & & \\
Enabling Factors & & \\
Income (\%) & 15.1 & 15.5 \\
< \$30,000 & 20.4 & 23.1 \\
\$30,000-\$59,999 & 23.0 & 20.7 \\
\$60,000-\$89,999 & 16.1 & 15.3 \\
\$90,000-\$119,999 & 10.9 & 11.0 \\
\$120,000-\$149,999 & 5.2 & 4.6 \\
\$150,000-\$179,999 & 9.0 & 9.8 \\
$>$ \$180,000 & & \\
Health Insurance (\%) & 86.9 & 84.1 \\
Yes & 13.1 & 15.9 \\
No & & \\
Education (\%) & 37.7 & 38.8 \\
Less than a Bachelor's Degree & 35.7 & 35.3 \\
Bachelor's Degree & 26.6 & 25.9 \\
More than a Bachelor's Degree & & \\
Illness Component & 29.7 & \\
Yes-Chronic Condition & 70.3 & \\
No-Healthy & & \\
\hline
\end{tabular}

\subsection{Procedure}

Potential participants were randomly selected and approached by research assistants in community areas around San Diego County. Participants were asked to complete a 5- to 10-minute survey, which they were told was for the purpose of investigating the factors associated with hiring an HCA. Participants were provided with a cover letter stating that they must be 18 years of age or older to participate, that all information provided would be kept anonymous and confidential, and that participation was voluntary and could be discontinued at any time. First, participants read a definition and description of an HCA and were asked how well they understood the role of an HCA. Participants were then asked to read a vignette and respond to a set of questions as though they were the person described in the vignette. In the last section of the survey, participants were asked to respond to various questions about themselves and how likely they would be to hire an HCA should they or their parents become ill or injured. When participants were finished with their surveys, they were given five dollars as a token of appreciation for their participation. 


\subsection{Measures}

The measures used to examine one's perceived likelihood of hiring an HCA and the predisposing, enabling, and illness factors are described below.

\subsubsection{Predisposing Factor}

The variables used to assess the predisposing factor were participant gender, age, ethnicity, and marital status. These demographic variables were assessed via a self-report questionnaire. Age was measured in years as a continuous variable. Ethnicity was assessed using the following categories: Caucasian, African American, Asian/Pacific Islander, American Indian, Latino/Hispanic/Mexican American, multi-ethnic, or other. However, the sample sizes for all non-Caucasian ethnicities were relatively small; for this reason, ethnicity was dichotomized into two groups: Caucasian and Minority. Participants who selected "multi-ethnic" were classified as a Minority. Marital status was classified as "single", "married/domestic partner", or "divorced/separated/widowed".

\subsubsection{Enabling Factor}

The variables used to assess the enabling factor were participant's total family income, health insurance status, and education level. Total family income was divided into seven categories (Table 1). Health insurance status was defined as participant's "yes" or "no" response when asked whether they had health insurance. Education was divided into three groups, those with less than a bachelor's degree (Less than high school, high school graduate, associate's/trade school), those with a bachelor's degree, and those with more than a bachelor's degree.

\subsubsection{Illness Component}

The illness component variable was measured dichotomously for the respondent, as presence of a chronic health condition or absence of a chronic health condition in the person for whom the HCA would be hired. That is, the participant was asked whether he/she had a chronic health condition or whether his/her parent had a chronic health condition. Participants also were asked whether they (or their parents) suffered from any medical conditions. These responses were coded as chronic or not, based on the Center for Disease Control's list of chronic conditions (CDC, 2014).

\subsubsection{Likelihood of Hiring a Healthcare Advocate}

Participants were asked to report how likely they would be to hire an HCA for themselves, or for a parent in the future, should they or their parent become ill or injured. This outcome variable was measured on a 10-point Likert scale, ranging from 1 (not at all likely) to 10 (extremely likely).

\subsection{Analyses}

Confirmatory factor analysis and structural equation modeling were used to test the effects of predisposing, enabling, and illness factors on the perceived likelihood of hiring a healthcare advocate for oneself or for a parent. First, the measurement model was tested to examine how well the measurement variables loaded onto the latent variable (e.g., insurance, income, and education loading onto one enabling factor variable).

Secondly, the full structural model was tested for both groups. Use of the chi-squared test to assess 
model fit has been deemed unsatisfactory for numerous reasons, including being an unreliable indicator when dealing with large sample sizes (Tanaka, 1993). Because of these limitations, many researchers (e.g., Hoyle, 2000; Tanaka, 1993) have suggested using multiple measures of model fit instead of just statistical fit. In the present study, the Comparative Fit Index (CFI; Bentler, 1990) and the root mean square error of approximation (RMSEA; Steiger, 1990) were used to interpret descriptive fit. Both the CFI and RMSEA are standardized measures of descriptive model fit that range in value from 0 to 1 . For the CFI, values greater than .95 indicate a reasonable model, and values greater than .90 indicate a plausible model. For the RMSEA, values less than .08 indicate acceptable model fit, and values less than .05 indicate good model fit.

\section{Results}

\subsection{Likelihood of Hiring an HCA for Self-Model Results}

A one-factor predisposing model was tested, using the confirmatory factor analysis procedure in EQS. The predisposing factor was indicated by four observed variables. This one-factor model fit well both statistically $\left(\chi^{2}[2, N=650]=4.319, p=.115\right)$ and descriptively $(\mathrm{CFI}=.986, \mathrm{RMSEA}=.043)$. All standardized factor loadings were moderate to large and statistically significant (values ranged from .340 to .836 ), except for gender (.016). Gender was removed from the predisposing factor because of its low and non-significant factor loading. The standardized factor loading remained significant with gender removed from the model (values ranged from .350 to .822 ).

Another confirmatory factor analysis was performed to test a one-factor enabling model. This enabling factor was indicated by three observed variables. Statistical and descriptive fit indexes could not be used to evaluate the enabling latent variable because the model was just-identified, meaning that there were zero degrees of freedom. Thus, the standardized factor loadings were used to determine model fit. All standardized factor loadings were moderate to large and statistically significant (values ranged from .449 to .700$)$.

Finally, a four-factor model predicting the likelihood of hiring an HCA was tested, using structural equation modeling in EQS. The predisposing and enabling latent variables were each indicated by three observed variables. The other two variables in the model were observed variables that represented illness level and likelihood of hiring a healthcare advocate. This model did not fit well statistically $\left(\chi^{2}\right.$ $[16, N=650]=31.095, p=.013)$, but it did fit well descriptively $(\mathrm{CFI}=.962$, RMSEA $=.040)$. All standardized factor loadings were generally large and statistically significant for both the predisposing (values ranged from .352 to .735) and enabling factors (values ranged from .465 to .682). The direct path predicting the enabling factors latent variable from the predisposing factors latent variable was statistically significant $(\beta=.550, p<.05$ ). Thus, predisposing factors (age, marital status, ethnicity) predicted enabling factors (education, income, education). Additionally, the indirect path predicting illness level with predisposing factors was statistically significant $(\beta=.296, p<.05)$. That is, predisposing factors were significantly related to an individual's health status. However, the paths from 
enabling factors to illness, from illness to the likelihood of hiring an HCA, and the other indirect paths shown in Figure 3 were not significant.

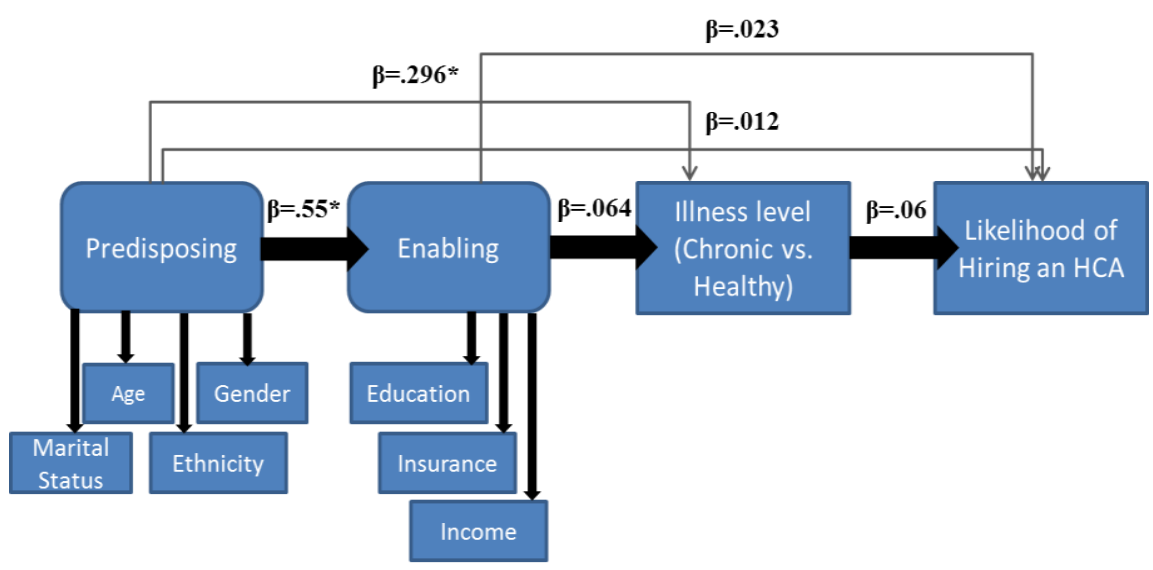

Figure 3. Path Loadings on Enabling, Illness Level and Likelihood of Hiring an HCA for Self

\subsection{Likelihood of Hiring an HCA for a Parent-Model Results}

A one-factor predisposing model was tested, using the confirmatory factor analysis procedure in EQS. The predisposing factor was indicated by four observed variables. This one-factor model did not fit well statistically $\left(\chi^{2}[2, N=1740]=7.854, p=.0197\right)$, but did fit well descriptively $(\mathrm{CFI}=.981$, RMSEA $=.054)$. All standardized factor loadings were moderate to large and statistically significant (values ranged from .291 to .879), except for gender (.025). Again, gender was removed from the predisposing factor because of this low and non-significant factor loading. The standardized factor loading remained significant with gender removed from the model (values ranged from .305 to .854). Another confirmatory factor analysis was performed to test a one-factor enabling model. The enabling factor was indicated by three observed variables. Statistical and descriptive fit indexes could not be used to evaluate the enabling latent variable because the model was just-identified, meaning that there were zero degrees of freedom. In this case, the standardized factor loadings were used to determine model fit. All standardized factor loadings were moderate to large and statistically significant (values ranged from .419 to .743$)$.

Lastly, a four-factor model predicting the likelihood of hiring an HCA was tested, using structural equation modeling in EQS. The predisposing and enabling latent variables were each indicated by three observed variables. The other two variables in the model were observed variables that represented illness level and the likelihood of hiring an HCA. This model did not fit well statistically $\left(\chi^{2}[16, N=\right.$ $1740]=33.875, p=.006)$, but it did fit well descriptively $($ CFI $=.963$, RMSEA $=.044)$. All standardized factor loadings were generally large and statistically significant for both the predisposing (values ranged from .309 to .793) and enabling factors (values ranged from .508 to .615). The direct path predicting the latent variable representing enabling factors with the latent variable representing predisposing factors was statistically significant $(\beta=.511, p<.05)$. Thus, predisposing factors (age, 
marital status, ethnicity) predicted enabling factors (education, income, education). Additionally, the indirect path predicting illness level with predisposing factors was statistically significant $(\beta=.472, p$ $<.05)$. That is, predisposing factors were significantly related to an individual's health status. However, the paths from enabling factors to illness, from illness to the likelihood of hiring an HCA, and the other indirect paths shown in Figure 4 were not significant.

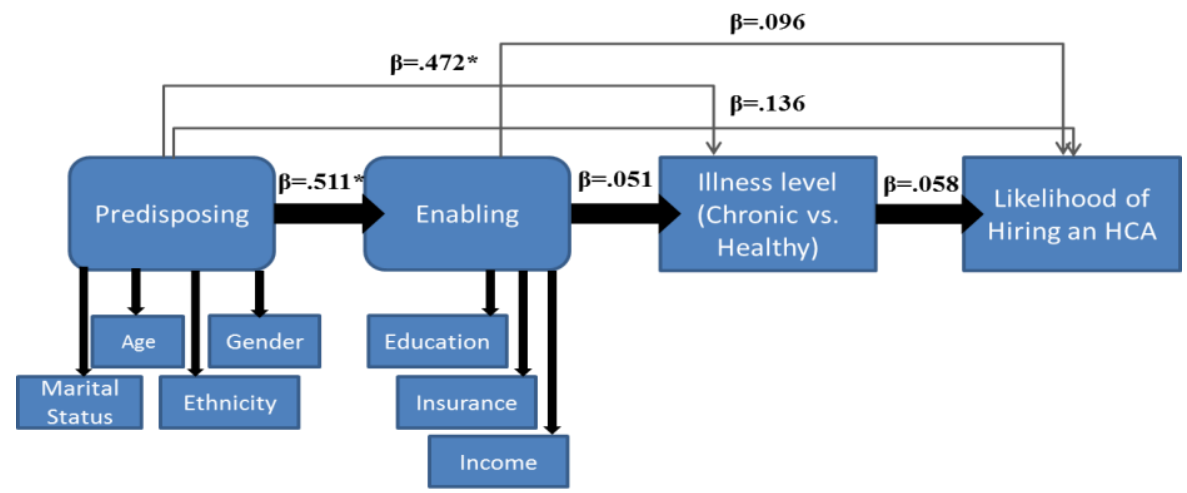

Figure 4. Path Loadings on Enabling, Illness Level and Likelihood of Hiring an HCA for a Parent

\section{Discussion}

In this study, an adaptation of the Andersen and Newman model was tested as a method to predict the perceived likelihood of hiring an HCA for one's self or an older parent. Two main hypotheses were tested. First, predisposing factors would be directly related to enabling factors, and enabling factors would be directly related to illness level, which would have a direct effect on the likelihood of hiring a healthcare advocate. The results partially supported the first hypothesis. Predisposing factors were, as predicted, significantly related to enabling factors. This was true for both the self and parent models. Therefore, an individual's age, ethnicity, and marital status collectively predicted income, education, and insurance status. However, enabling factors did not predict illness status, nor did illness status have a direct effect on the perceived likelihood of hiring an HCA. This could be a result of a limitation of the illness status variable that was used. Participants' responses were dichotomized into either "healthy" or as having a chronic condition on the CDC's list of chronic conditions. However, more detailed information about the self-reported illness, such as duration and severity, could create a more robust illness variable. Although the relationship was not specified in the hypothesized model, predisposing factors were also significantly related to illness status. This finding is supported by epidemiological research, which has identified demographic factors as contributors to health (Yusuf, Reddy, Ôunpuu, \& Anand, 2001; Katz, McHorney, \& Atkinson, 2000).

It was also hypothesized that the overall model depicted in Figure 2 would fit well both statistically and descriptively. This hypothesis was partially supported. For both predicting the perceived likelihood of hiring an HCA for oneself and of hiring for a parent, the adapted model did not fit well statistically, but did fit well descriptively. Limitations of the chi-squared test with regard to large sample sizes provide a 
strong explanation as to why the model did not fit well statistically. The model did fit well descriptively as a whole for both groups; however, upon further investigation of the path coefficients, only the path between predisposing and enabling factors was significant, and may have been driving the significance of the overall model.

Finally, we intended to compare the application of the proposed model for predicting the perceived likelihood of hiring an HCA for oneself and for a parent; however, the two groups could not be statistically compared because the individual model pathways were not significant. Despite this limitation, similar results and trends were found for both groups. For example, the relationships between predisposing factors and enabling factors, as well as between predisposing factors and illness level, were significant for both groups. This may suggest that there is not a difference in predicting additional care, whether it is for an individual or for a proxy such as a parent. This conclusion is consistent with previous research that found proxy ratings to be reasonably accurate and to have significant implications for the type of care and treatment provided (Essen, 2004). In addition, Bigatti et al. (2007) found moderate overall agreement on quality of well-being between patient and proxy ratings of women with fibromyalgia. This overall trend for predicting the perceived likelihood of hiring an HCA for oneself or a parent suggests that similar factors may influence the decision to hire an HCA, whether for oneself or a proxy.

HCAs are a new concept to many; therefore, it is important to understand the variables that may lead someone to hire an HCA, as well as in which populations and under what circumstances they may be most beneficial. With the baby boomers becoming seniors, the rates of dementia are increasing, and the number of family caregivers will also increase (Knickman \& Snell, 2002; Lynn \& Adamson, 2003). Health concerns and illnesses that are more prevalent in older adults, such as chronic diseases and dementia, require consistent healthcare and place a heavy economic burden on the healthcare system (Anderson \& Horvath, 2004). Considering the complexity of the healthcare system, and thus of patient decision-making, it is important to offer services to assist people with chronic conditions that require long-term care. HCAs have the potential to help patients and their caregivers navigate the system, aid them in decision-making, and increase their overall quality of care.

Some limitations should be noted in the interpretation of the present results. Depending on the population or type of health-related service, the relationships between predisposing factors, enabling factors, illness factors, and health behavior may vary. Additionally, the definition and measurement of the major factors in the Andersen and Newman model were adapted to fit this particular study, and some variables had to be modified or excluded. Finally, the specific type of healthcare advocacy service examined in this study is a new service, with which many participants may not have been familiar. The brief description of an HCA that participants were asked to read before completing the survey may not have fully captured every aspect of the services that an HCA could provide. If participants were unclear as to the variety of services offered, it may explain why no difference was found in participants' responses for themselves versus for a parent. 


\section{Conclusion}

The Andersen and Newman model has been used and adapted to fit a variety of health behavior models. Future researchers should incorporate other constructs that might be important in understanding a person's likelihood of using an HCA, such as the inclusion of social structure, health beliefs, or community variables. Because this was the first model comparison of the likelihood of hiring an HCA for two separate groups, future researchers should investigate whether these findings are similar or different for other populations, such as hiring for a child, for different ethnic groups, or for differing levels of socioeconomic status. Exploring any disparities that may exist between groups has the potential to help the healthcare system, health providers, and policy makers reach and serve patients who would benefit the most from healthcare advocacy services. Successfully targeting these groups could reduce overall cost and the burden on the healthcare system, as well as increasing quality of care and quality of life in these patients.

\section{References}

Aday, L. A., \& Awe, W. C. (1997). Health services utilization models. Handbook of health behavior research I: Personal and social determinants, 153-172.

Andersen, R., \& Newman, J. F. (1973). Societal and individual determinants of medical care utilization in the United States. Milbank Quarterly, 83(4). https://doi.org/10.2307/3349613

Anderson, G., \& Horvath, J. (2004). The growing burden of chronic disease in America. Public health reports, 119(3), 263. https://doi.org/10.1016/j.phr.2004.04.005

Bentler, P. M. (1990). Comparative fit indexes in structural models. Psychological bulletin, 107(2), 238. https://doi.org/10.1037/0033-2909.107.2.238

Bigatti, S. M., Cronan, T. A., Frederick, H., \& Kaplan, R. M. (2007). Spouses of women with the fibromyalgia syndrome as proxy raters of health status. Journal of Musculoskeletal Pain, 15(3), 9-17. https://doi.org/10.1300/J094v15n03_03

Bodenheimer, T. (2008). Coordinating care-A perilous journey through the healthcare system. New England Journal of Medicine, 358, 1064-1071. https://doi.org/10.1056/NEJMhpr0706165

Boyd, C. M., Boult, C., Shadmi, E., Leff, B., Brager, R., Dunbar, L., Wolff, J., \& Wegener, S. (2007). Guided care for multimorbid older adults. The Gerontologist, 47(5), 697-704. https://doi.org/10.1093/geront/47.5.697

Carlson, J. A., Imberi, J. E., Cronan, T. A., Villodas, M. T., Brown, K. C., \& Talavera, G. A. (2011). Factors Related to the Likelihood of Hiring a Health Advocate. Californian Journal of Health Promotion, 9(1).

Center for Disease Control (CDC). (2014, December 31). Chronic Disease Prevention and Health Promotion. Retrieved from http://www.cdc.gov/chronicdisease/

Cousin, G., Mast, M. S., Roter, D. L., \& Hall, J. A. (2012). Concordance between physician communication style and patient attitudes predicts patient satisfaction. Patient education and 
counseling, 87(2), 193-197. http://doi.org/10.1016/j.pec.2011.08.004

Cronan, T. A., Carlson, J. A., Imberi, J., Villodas, M., Vasserman-Stokes, E., \& Dowell, A. (2010). The effects of social support and confidence in the healthcare system on the likelihood of hiring a health advocate. Psychology research and behavior management, 3, 41. https://doi.org/10.2147/PRBM.S9149

Elwyn, G., Frosch, D., Thomson, R., Joseph-Williams, N., Lloyd, A., Kinnersley, P., ... Barry, M. (2012). Shared decision making: A model for clinical practice. Journal of general internal medicine, 27(10), 1361-1367. https://doi.org/10.1007/s11606-012-2077-6

Essen, L. V. (2004). Proxy ratings of patient quality of life factors related to patient-proxy agreement. Acta Oncologica, 43(3), 229-234. https://doi.org/10.1080/02841860410029357

Family Caregiver Alliance. (2012). Selected Caregiver Statistics. Retrieved from https://www.caregiver.org/selected-caregiver-statistics

Fani Marvasti, F., \& Stafford, R. S. (2012). From Sick Care to Healthcare-Reengineering Prevention into the US System. New England Journal of Medicine, 367(10), 889-891. https://doi.org/10.1056/NEJMp1206230

Frostholm, L., Fink, P., Christensen, K. S., Toft, T., Oernboel, E., Olesen, F., \& Weinman, J. (2005). The patients' illness perceptions and the use of primary healthcare. Psychosomatic Medicine, 67(6), 997-1005. https://doi.org/10.1097/01.psy.0000189164.85653.bc

Gaskin, D. J., Dinwiddie, G. Y., Chan, K. S., \& McCleary, R. (2011). Residential segregation and disparities in healthcare services utilization. Medical Care Research and Review.

Gelberg, L., Andersen, R. M., \& Leake, B. D. (2000). The Behavioral Model for Vulnerable Populations: Application to medical care use and outcomes for homeless people. Health services research, 34(6), 1273.

Hoyle, R. H. (Ed.). (2000). Structural equation modeling: Concepts, issues, and applications. Sage Publications.

Hu, X., \& Stowe, C. J. (2013). The Effect of Income on Health Choices: Physical Activity and Alcohol Use. In 2013 Annual Meeting, August 4-6, 2013, Washington, DC. Agricultural and Applied Economics Association.

Hurst, M. (2007). Defining the field: Health Advocacy. Sarah Lawrence College. Retrieved From http://www.slc.edu/public-health-advocacy/Defining_the_Field.html

Kaplan, R. M. (2004). Shared medical decision making: A new tool for preventive medicine. American journal of preventive medicine, 26(1), 81-83. https://doi.org/10.1016/j.amepre.2003.09.022

Katz, D. A., McHorney, C. A., \& Atkinson, R. L. (2000). Impact of Obesity on Health-related Quality of Life in Patients with Chronic Illness. Journal of General Internal Medicine, 15(11), 789-796. https://doi.org/10.1046/j.1525-1497.2000.90906.x

Knickman, J. R., \& Snell, E. K. (2002). The 2030 problem: Caring for aging baby boomers. Health services research, 37(4), 849-884. https://doi.org/10.1034/j.1600-0560.2002.56.x 
Lodi-Smith, J., Jackson, J., Bogg, T., Walton, K., Wood, D., Harms, P., \& Roberts, B. W. (2010). Mechanisms of health: Education and health-related behaviours partially mediate the relationship between conscientiousness and self-reported physical health. Psychology and Health, 25(3), 305-319. https://doi.org/10.1080/08870440902736964

Lynn, J., \& Adamson, D. M. (2003). Living well at the end of life. Adapting healthcare to serious chronic illness in old age. RAND CORP SANTA MONICA CA.

Neumann, P. J., Araki, S. S., \& Gutterman, E. M. (2000). The use of proxy respondents in studies of older adults: Lessons, challenges, and opportunities. Journal of the American Geriatrics Society, 48(12), 1646-1654. https://doi.org/10.1111/j.1532-5415.2000.tb03877.x

Parekh, A. K., \& Barton, M. B. (2010). The challenge of multiple comorbidity for the US healthcare system. Jama, 303(13), 1303-1304. https://doi.org/10.1001/jama.2010.381

Pickard, A. S., \& Knight, S. J. (2005). Proxy evaluation of health-related quality of life: A conceptual framework for understanding multiple proxy perspectives. Medical care, 43(5), 493. https://doi.org/10.1097/01.mlr.0000160419.27642.a8

Robinson, A., \& Thomson, R. (2001). Variability in patient preferences for participating in medical decision making: Implication for the use of decision support tools. Quality in Healthcare, 10 (suppl 1), i34-i38. https://doi.org/10.1136/qhc.0100034

Schoen, C., Osborn, R., Doty, M. M., Bishop, M., Peugh, J., \& Murukutla, N. (2007). Toward higher-performance health systems: Adults' healthcare experiences in seven countries, 2007. Health Affairs, 26, w717-w734. https://doi.org/10.1377/hlthaff.26.6.w717

Schulz, R., Cook, T. B., Beach, S. R., Lingler, J. H., Martire, L. M., Monin, J. K., \& Czaja, S. J. (2013). Magnitude and causes of bias among family caregivers rating Alzheimer disease patients. The $\begin{array}{lllll}\text { American Journal of } & \text { Geriatric }\end{array}$ https://doi.org/10.1016/j.jagp.2012.10.002

Schwartz, L. (2002). Is there an advocate in the house? The role of healthcare professionals in patient advocacy. Journal of medical ethics, 28(1), 37-40. https://doi.org/10.1136/jme.28.1.37

Singer, S. J., Burgers, J., Friedberg, M., Rosenthal, M. B., Leape, L., \& Schneider, E. (2011). Defining and measuring integrated patient care: Promoting the next frontier in healthcare delivery. Medical Care Research and Review, 68(1), 112-127. https://doi.org/10.1177/1077558710371485

Siu, A. L., Spragens, L. H., Inouye, S. K., Morrison, R. S., \& Leff, B. (2009). The ironic business case for chronic care in the acute care setting. Health Affairs, 28(1), 113-125. https://doi.org/10.1377/hlthaff.28.1.113

Steiger, J. H. (1990). Structural model evaluation and modification: An interval estimation approach. Multivariate behavioral research, 25(2), 173-180. https://doi.org/10.1207/s15327906mbr2502_4

Tanaka, J. S. (1993). Multifaceted conceptions of fit in structural equation models. Sage Focus Editions, $154,10$.

Van Liew, C., \& Cronan, T. A. (2012). Caring for a loved one with Alzheimer's Disease: Views from 
the population about hiring a healthcare advocate. American journal of Alzheimer's disease and other dementias, 27(8), 579-583. https://doi.org/10.1177/1533317512459795

Vogeli, C., Shields, A. E., Lee, T. A., Gibson, T. B., Marder, W. D., Weiss, K. B., \& Blumenthal, D. (2007). Multiple chronic conditions: Prevalence, health consequences, and implications for quality, care management, and costs. Journal of general internal medicine, 22(3), 391-395. https://doi.org/10.1007/s11606-007-0322-1

Wagner, E. H., Austin, B. T., Davis, C., Hindmarsh, M., Schaefer, J., \& Bonomi, A. (2001). Improving chronic illness care: Translating evidence into action. Health Affairs, 20, 64-78. https://doi.org/10.1377/hlthaff.20.6.64

Wilber, K. H., Allen, D., Shannon, G. R., \& Alongi, S. (2003). Partnering Managed Care and Community-Based Services for Frail Elders: The Care Advocate Program. Journal of the American Geriatrics Society, 51(6), 807-812. https://doi.org/10.1046/j.1365-2389.2003.51257.x

Wright, K. B., Sparks, L., \& O'Hair, H. D. (2012). Health communication in the 21st century. John Wiley \& Sons.

Yang, Z., Norton, E. C., \& Stearns, S. C. (2003). Longevity and healthcare expenditures the real reasons older people spend more. The Journals of Gerontology Series B: Psychological Sciences and Social Sciences, 58(1), S2-S10. https://doi.org/10.1093/geronb/58.1.S2

Yusuf, S., Reddy, S., Ôunpuu, S., \& Anand, S. (2001). Global burden of cardiovascular diseases part I: General considerations, the epidemiologic transition, risk factors, and impact of urbanization. Circulation, 104(22), 2746-2753. https://doi.org/10.1161/hc4601.099487 